\title{
Tumor neuroectodérmico primitivo primario de páncreas en un paciente pediátrico. Reporte de caso y revisión de la literatura
}

\section{RESUMEN}

Los tumores neuroectodérmicos primitivos son tumores de células pequeñas y redondas derivadas del tejido blando, pertenecen a la familia del sarcoma de Ewing. El tumor neuroectodérmico primario del páncreas es muy poco frecuente, en la literatura sólo se han reportado 17 casos. Reportamos el caso de una paciente de 9 años de edad, con diagnóstico de tumor neuroectodérmico primario del páncreas a la que se le realizó una cirugía de Whipple e inició tratamiento de quimioterapia con ciclofosfamida, vincristina, doxorrubicina pegilada, etopósido e ifosfamida y radioterapia. Desde marzo de 2013 la paciente se encuentra en vigilancia, con un periodo de seguimiento de 23 meses. Actualmente, se encuentra en buenas condiciones generales y sin evidencia de actividad tumoral.

Palabras clave: tumor neuroectodérmico primitivo, tumor neuroectodérmico primitivo primario de páncreas, procedimiento de Whipple.

\section{Primitive neuroectodermal tumor of the pancreas. Case report and review of the literature}

\begin{abstract}
The primitive neuroectodermal tumor of the pancreas, are small round cell tumors arising from the soft tissue that belongs to the family of Ewing's sarcoma, The primary neuroectodermal (PNET) of the pancreas is very rare, have been reported only 17 cases in the literature. We report the case of a 9 year-old female patient, with PNET of the pancreas, was carried out a Whipple surgery, and began chemotherapy treatment with, vincristine, doxorubicin Pegylated, etoposide and ifosfamide and radiation therapy. Currently, the patient is in monitoring since March 2013, with a follow up of 23 months, overall in good condition and without evidence of tumor activity.
\end{abstract}

Key words: Primitive Neuroectodermal tumor, Primitive neuroectodermal tumor of the pancreas, Whipple procedure.

\author{
Lizbeth Carolina Riveros-Ramos ${ }^{1}$ \\ Liliana Velasco-Hidalgo ${ }^{2}$ \\ Jaime Shalkow-Klincovstein ${ }^{3}$ \\ César Mauricio Rojas-Maruri ${ }^{4}$ \\ Rocío Cárdenas-Cardos ${ }^{2}$ \\ Roberto Rivera-Luna ${ }^{5}$ \\ ${ }^{1}$ Residente de Pediatría. \\ ${ }^{2}$ Servicio de Oncología. \\ ${ }^{3}$ Instituto Nacional para la Salud de la Infancia y \\ Adolescencia. \\ ${ }^{4}$ Departamento de Patología. \\ ${ }^{5}$ Subdirección de Hemato-Oncología. \\ Instituto Nacional de Pediatría, México.
}

Recibido: 6 de enero del 2015

Aceptado: 10 de septiembre del 2015

Correspondencia: Dra. Liliana Velasco-Hidalgo Servicio de Oncología Instituto Nacional de Pediatría Insurgentes Sur 3700-C CP 04530 México, D.F. lilianavh@hotmail.com

Este artículo debe citarse como Riveros-Ramos LC, Velasco-Hidalgo L, ShalkowKlincovstein J, Rojas-Maruri CM, Cárdenas-Cardos R, Rivera-Luna R. Tumor neuroectodérmico primitivo primario de páncreas en un paciente pediátrico. Reporte de caso y revisión de la literatura. Acta Pediatr Mex 2016;37:26-31. 


\section{INTRODUCCIÓN}

El sarcoma de Ewing es la segunda neoplasia ósea maligna más frecuente en pediatría; sin embargo, también puede presentarse en tejidos blandos. ${ }^{1}$ Los tumores neuroectodérmicos primarios son tumores de células pequeñas y redondas derivadas del tejido blando, pertenecen a la familia del sarcoma de Ewing., ${ }^{2,3}$ Estas neoplasias exhiben un fenotipo neural, expresan la proteína MIC2- (CD99) y 85\% de los casos muestra translocación cromosómica t(11:22) (q24; q12). ${ }^{4,5}$ El tumor neuroectodérmico primario puede presentarse en órganos sólidos como riñón, pulmón, ovario, vejiga y páncreas, entre otros. ${ }^{5,6} \mathrm{El}$ tumor neuroectodérmico primario del páncreas es un tumor muy poco frecuente que habitualmente se presenta en niños o adultos jóvenes. ${ }^{7-9}$ En la literatura sólo se han reportado 17 casos. $^{9-11}$ En este artículo se presentan las características clínicas, el tratamiento y la evolución de un paciente pediátrico con tumor neuroectodérmico primario del páncreas, así como una revisión de la literatura.

\section{PRESENTACIÓN DEL CASO}

Niña de 9 años de edad proveniente del estado deTabasco. Inició su padecimiento con ictericia, palidez, astenia, adinamia, anemia, pérdida de peso de 3 kilogramos en tres meses y dolor abdominal epigástrico, por lo que se inició protocolo de estudio realizando una tomografía y una resonancia magnética que mostraron un tumor retroperitoneal de $14 \times 10 \times 8 \mathrm{~cm}$, localizado en la cabeza del páncreas. Se tomó una biopsia percutánea que reportó una neoplasia maligna de células pequeñas y se le envió a nuestro centro, el Instituto Nacional de Pediatría. A su ingreso se encontró, en la exploración física, dolor epigástrico de tipo cólico de intensidad variable, irradiado a la región lumbar, hepatomegalia de $3 \mathrm{~cm}$ por debajo del borde costal, melena, sin evidencia de tumores o ganglios. Se hicieron los siguientes estudios de laboratorio: bilirrubina total: $0.26 \mathrm{mg} / \mathrm{dL}$; aspartato-aminotransferasa: $74 \mathrm{UI} / \mathrm{L}$; alanina-aminotransferasa $121 \mathrm{UI} / \mathrm{L}$, fosfatasa alcalina $245 \mathrm{UI} / \mathrm{L}$, lactato-deshidrogenasa 208, gamma-glutamiltranspeptidasa $196 \mathrm{UI} / \mathrm{L}$, amilasa $1.1 \mathrm{UI} / \mathrm{L}$.

Se le practicó cirugía de Whipple con resección completa de un tumor en la cabeza del páncreas, de $14 \times 10 \times 8 \mathrm{~cm}$ en sus diámetros mayores y $270 \mathrm{~g}$ de peso (Figura 1), que infiltraba al duodeno, grasa y ganglios linfáticos peripancreáticos; así mismo se realizó colecistectomía. La cirugía tuvo una duración de 7 horas. No hubo complicaciones transoperatorias y el sangrado quirúrgico fue mínimo. La paciente ingresó estable a la unidad de cuidados intensivos.

El reporte histopatológico confirmó un tumor neuroectodérmico primitivo/sarcoma de Ewing, en la cabeza del páncreas, con menos de 10\% de necrosis y calcificaciones focales, con infiltración al duodeno, conducto de Wirsung y tejidos blandos peripancreáticos, así como a uno de cuatro ganglios locorregionales. No se encontró actividad en los márgenes quirúrgicos de la resección (Figuras 2-4). Al quinto día de posoperatorio la paciente desarrolló choque séptico, insuficiencia renal y salida de material fecaloide

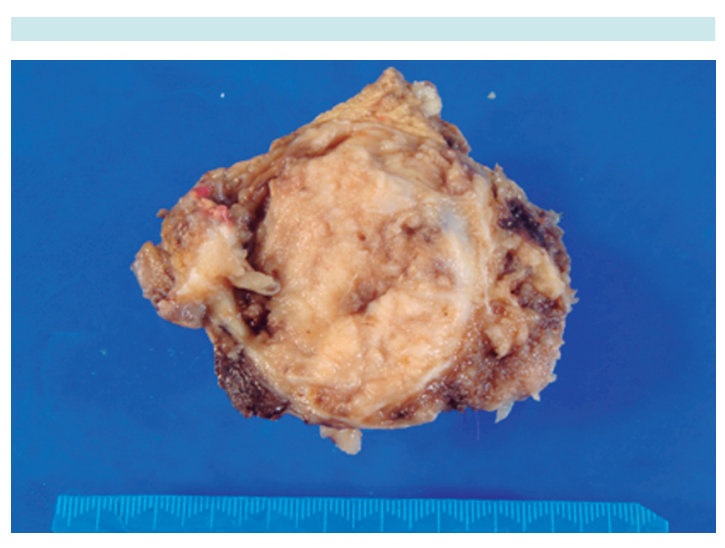

Figura 1. Pieza quirúrgica producto de la resección de un tumor en la cabeza del páncreas y segmento de duodeno; dimensiones $14 \times 10 \times 8 \mathrm{~cm}$; peso $270 \mathrm{~g}$. 


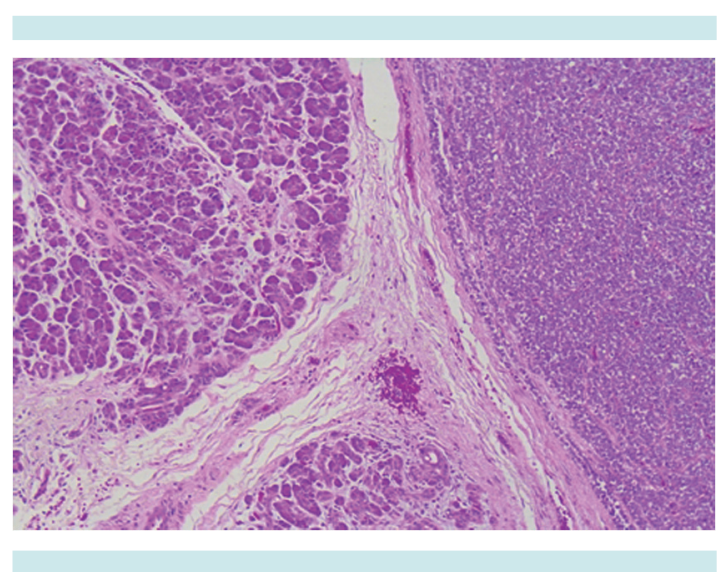

Figura 2. Fotomicrografía: ácinos pancreáticos (izquierda) en íntima relación con una neoplasia de células pequeñas redondas y azules (derecha).

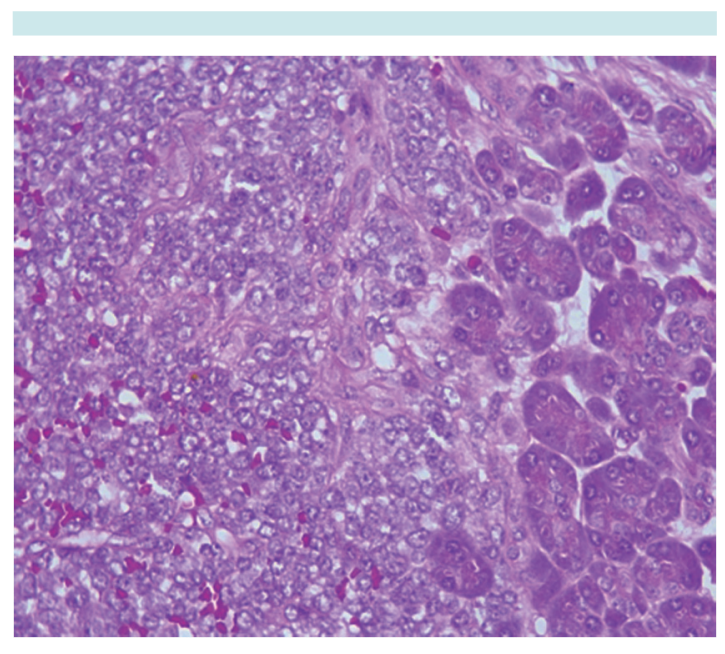

Figura 3. Fotomicrografía: la neoplasia infiltra los ácinos pancreáticos.

por la herida quirúrgica, por lo que tuvo que ser sometida a una segunda laparotomía por sepsis abdominal donde se evidenció dehiscencia de la anastomosis yeyuno-pancreática. Se lavó la cavidad, se realizó necrosectomía y una nueva anastomosis con colocación de drenajes. Posteriormente la paciente tuvo fístula biliocutánea que fue tratada con sistema de presión negativa y drenaje percutáneo, así como tratamiento médico con octreotida y tratamiento médico de

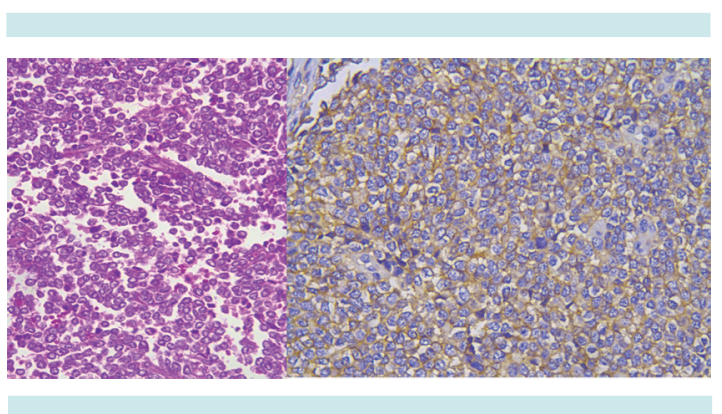

Figura 4. Fotomicrografía: características morfológicas. Células pequeñas redondas y azules (izquierda) positivas para la tinción de inmunohistoquimica CD99 (derecha); junto con otras tinciones apoya el diagnóstico de tumor neuroectodérmico primitivo/sarcoma de Ewing.

la sepsis. La paciente requirió antibióticos de amplio espectro y la complicación se resolvió.

En el tumor primario se realizaron los marcadores de inmunohistoquímica referidos en el Cuadro 1. Como parte de la extensión de la neoplasia maligna se realizaron los siguientes estudios para evaluar la extensión de la enfermedad, establecer un plan de tratamiento y hacer un pronóstico: tomografía de pulmón de alta resolución, gammagrama óseo y biopsia bilateral de médula ósea, éstos se reportaron negativos para metástasis.

Tres meses después de la cirugía, y una vez que se habían resuelto las complicaciones quirúr-

Cuadro 1. Inmunohistoquímica

\begin{tabular}{lc}
\hline Marcador & Resultado \\
CD99 & Positivo \\
Bcl-2 & Positivo \\
Vimentina & Positivo \\
Sinaptofisina & Positivo (focalmente) \\
K67 & Positivo (más de 50\%) \\
CD10 & Negativo \\
CD45 & Negativo \\
EMA & Negativo \\
CK AE1/AE3 & Negativo \\
Cromogranina & Negativo \\
TdT & Negativo
\end{tabular}


gicas, se inició el tratamiento de quimioterapia con base en el Protocolo Nacional para sarcoma de Ewing con ciclofosfamida $\left(2.1 \mathrm{gr} / \mathrm{m}^{2}\right.$ al día durante dos días), vincristina $\left(2 \mathrm{mg} / \mathrm{m}^{2}\right.$ por 1 día), doxorrubicina pegilada $\left(50 \mathrm{mg} / \mathrm{m}^{2}\right.$ por 1 día) alternado con etopósido $\left(100 \mathrm{mg} / \mathrm{m}^{2}\right.$ al día durante 5 días) e ifosfamida ( $2 \mathrm{~g} / \mathrm{m}^{2}$ al día durante 5 días) por 8 ciclos y radioterapia en hemiabdomen superior, 50.4 Gy en 30 sesiones. ${ }^{11}$ Durante el tiempo que la paciente recibió quimioterapia presentó 4 cuadros de neutropenia y fiebre, los cuales se resolvieron sin complicaciones. Actualmente la paciente se encuentra bajo vigilancia, desde marzo del 2013, en buenas condiciones generales y sin evidencia de actividad tumoral.

\section{ANÁLISIS CUALITATIVO DE LA LITERATURA}

Se realizó una búsqueda exhaustiva en las siguientes bases: Medline 1950-2013 (OVID), Embase 1980-2013 (OVID), LILACS (1998 a 2013), ARTEMISA (1999 a 2013) y SCIELO (1999 a 2013). Se encontraron 9 artículos en los cuales se reportaban casos de pacientes con tumor neuroectodérmico primitivo/sarcoma de Ewing en páncreas. Movahedi, en 2002, reportó 7 casos, sin embargo, sólo uno se presentó en población pediátrica. De los 16 casos encontrados sólo 7 fueron en pacientes pediátricos con una media de edad de 10.8 años. Se describen las características clínicas, tratamiento y evolución de los pacientes en el Cuadro 2.

\section{DISCUSIÓN}

El sarcoma de Ewing es el segundo tumor maligno primario de hueso más común en pediatría, precedido por el osteosarcoma. Sin embargo, el sarcoma de Ewing también puede afectar tejidos blandos..$^{2,7}$ La histología característica de estos tumores son células pequeñas, redondas y azules; es común en niños, adolescentes y adultos jóvenes. ${ }^{1,5}$ Se considera que no hay diferencia histológica entre los tumores de esta familia., ${ }^{2,8}$
Los tumores de páncreas son extremadamente raros en niños. ${ }^{7,5}$ Los tumores neuroectodérmicos primitivos son histológicamente indistinguibles de los del tipo óseo y se han documentado en otros sitios como vagina, septo retrovaginal, intestino delgado, ovarios, próstata, esófago y riñón. ${ }^{5,3}$ En la literatura médica internacional hay muy pocos casos registrados. En 2013 se habían reportado sólo 17 casos en los que se describían las manifestaciones más comunes al momento del diagnóstico. ${ }^{4}$ En $85 \%$ de los casos el dolor abdominal fue el signo más importante de la enfermedad; con menor frecuencia se reportaron vómito, fiebre y pérdida de peso, como en el caso de la paciente que presentamos. Sólo se reportaron 7 casos pediátricos (en menores de 18 años).

El tratamiento de estos pacientes no está bien establecido debido a la baja frecuencia de la enfermedad. De los casos reportados 14.2\% fueron tratados únicamente con cirugía, 14.2\% con quimioterapia y radioterapia y $57.1 \%$ con quimioterapia y cirugía, dependiendo de la extensión de la enfermedad. En uno de los casos no se reportó el tratamiento utilizado. En todos los casos el tratamiento inicial fue la cirugía de Whipple (pancreatoduodenectomía). Internacionalmente, en pacientes con sarcoma de Ewing sin especificar su localización, se recomienda el uso de quimioterapia con las mismas drogas que empleamos en el Protocolo Nacional, basado en el protocolo Euroewing 99, que ha mostrado ser bien tolerado y logra una buena respuesta. ${ }^{11}$ Nuestra paciente recibió tratamiento con vincristina, ciclofosfamida y doxorrubicina, alternado con ifosfamida y etopósido; además de radioterapia abdominal por enfermedad ganglionar. Este tratamiento ha sido bien tolerado y se ha reportado supervivencia de hasta $70 \%$ de los casos en series internacionales. De los 7 pacientes pediátricos reportados en la literatura $5(71.4 \%)$ se encuentra vivos. Nuestra paciente está viva y sin datos de actividad tumoral en un seguimiento que dura ya 48 meses. 


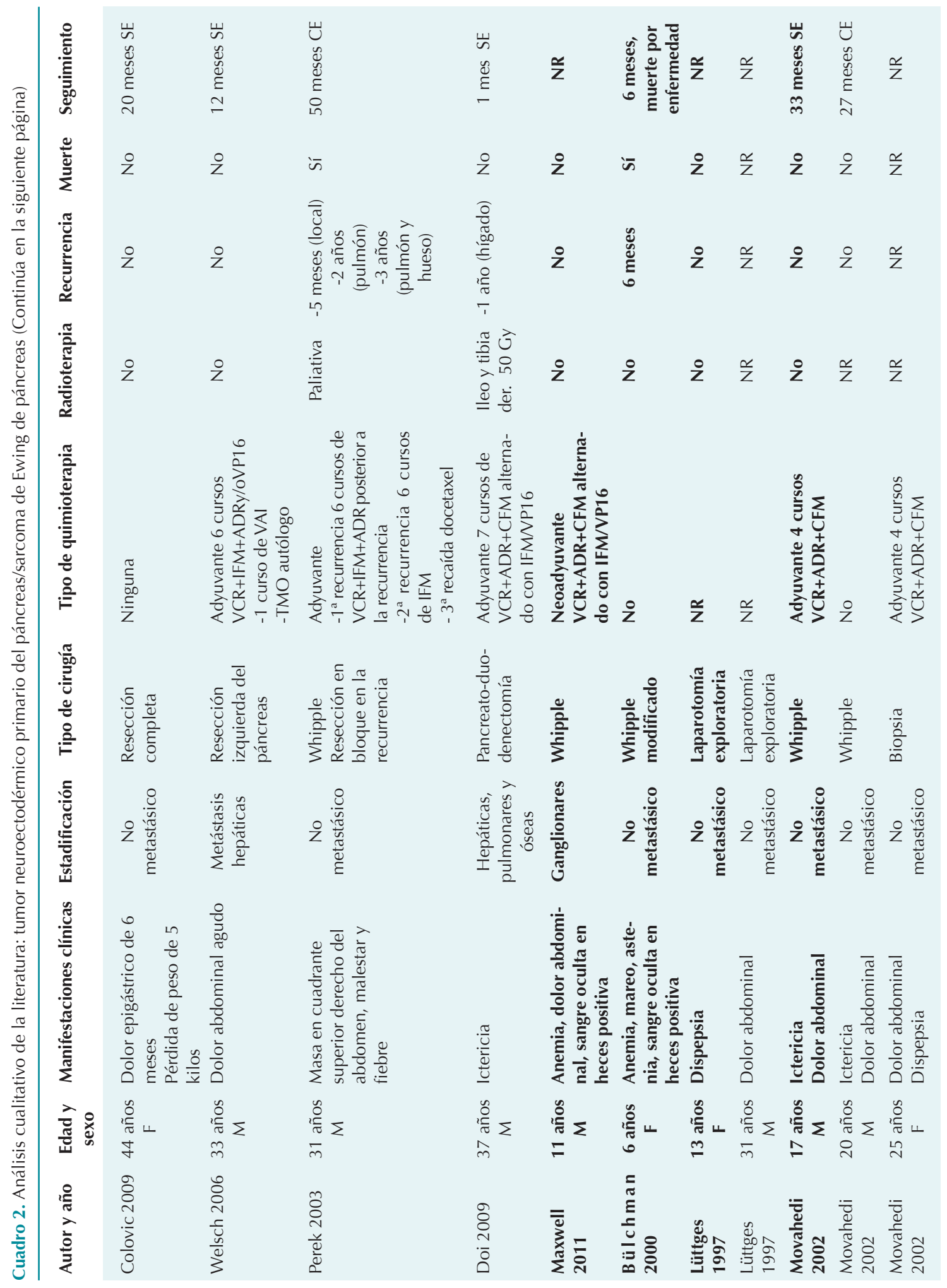


Riveros-Ramos LC et al. Tumor neuroectodérmico primitivo primario de páncreas

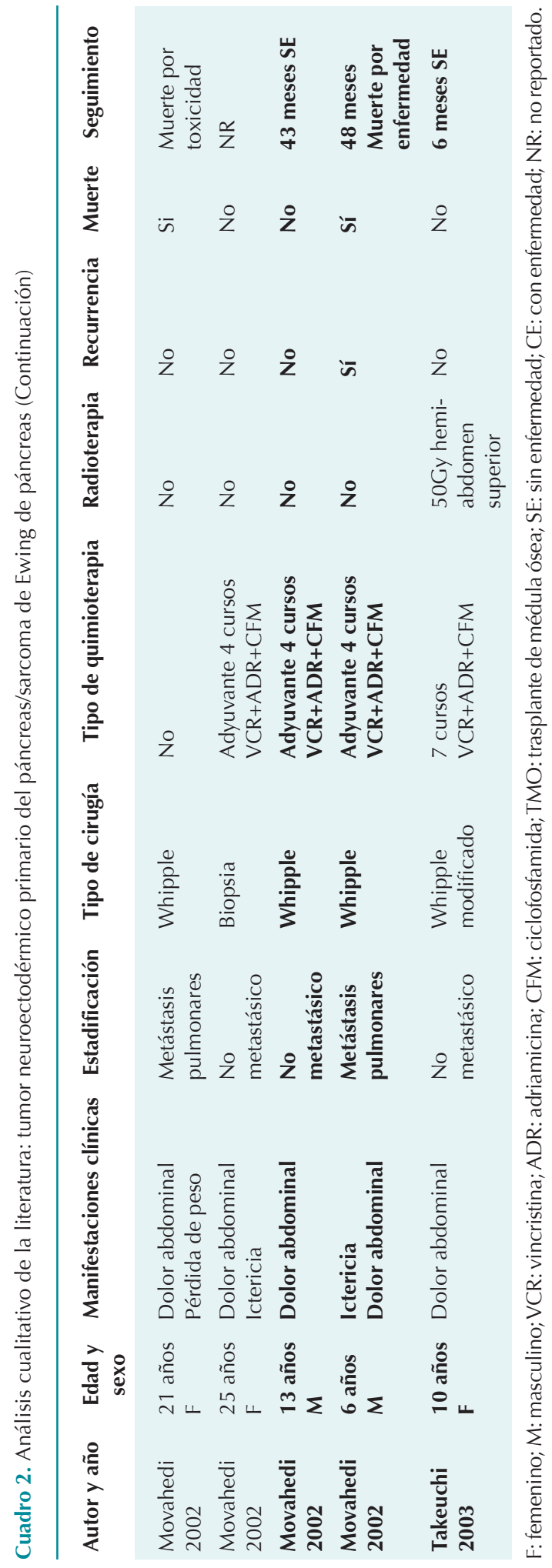

Es importante considerar que $85 \%$ de los pacientes con sarcoma de Ewing presenta la translocación $\mathrm{t}(11,22)(\mathrm{q} 24 ; \mathrm{q} 12)$. En nuestra paciente no evaluamos esa alteración pero, actualmente, el diagnóstico integral de estos pacientes incluye la determinación de alteraciones cromosómicas, por lo que es necesario integrar esas pruebas al diagnóstico de los pacientes en México.

\section{REFERENCIAS}

1. Doi H, Ichikawa S, Hiraoka A, et al. Primitive neuroectodermal tumor of the pancreas. Intern Med 2009;48:329-333.

2. Maxwell L, Hederman A, Jackson C, et al. Uncommon presentation of rare disorder-duodenal ulcer secondary to invasive pancreatic primitive neuroectodermal tumor: case report and review of the literature. J Pediatr Hematol Oncol 2011;33(7):543-8.

3. Bulchmann G, Schuster T, Hass RJ, et al. Primitive Neuroectodermal tumor of the páncreas. An extremely rare tumor. Case report and review of the literature. Klin Pediatr 2000;212:185-188.

4. Luttges J, Pierre E, Zamboni G, Weh G, Lietz H, Kussmann J, Kloppel G. Malignant non-epithelial tumors of the pancreas. Pathologe 1997;18:233-237.

5. Welsch T, Mechtersheimer G, Aulman S, et al. Huge primitive neuroectodermal tumor of the pancreas: report of a case and review of the literature. World J Gastroenterol 2006;12:6070-6073.

6. Colvic RB, Grubor NM, Micev MT, Matic SV, et al. Perigastric extraskeletal Ewing's sarcoma: A Case report. World J Gastroenterol 2009;14:245-247.

7. Gallegos-Castorena S, Cárdenas-Cardos R. 2010. Sarcoma de Ewing y tumor neuroectodérmico primitivo: Protocolos técnicos cáncer en niños. México, D.F.: Editores de textos mexicanos S.A. de C.V. pp. 219-233.

8. Movahedi-Lankarani S, Hurban RH, Westra WH et al Primitive neuroectodermal tumors of the pancreas. A report of seven cases of a rare neoplasm. Am J Surg Phatol 2001;26:1040-1047.

9. Tekeuchi M, Kuwae Y, Hamana K, et al. Primitive neuroectodermal tumor of the pancreas. Arch Histopathology D. 2003;10:23-26.

10. Prithviraj Bose, Paari Murugan, Elizabeth Gillies, Jennifer L. Holter. Extraosseous Ewing's sarcoma of the pancreas. Int J Clin Oncol 2012;17(4):399-406.

11. Perek S, Perek A, Sarman K et al Primitival neuroectodermal tumor of the pancreas. A case report of an extremely rare tumor. Pancreatology 2003;2:352-356. 